\title{
Aspectos microbiológicos e de qualidade do leite bovino ${ }^{1}$
}

\author{
Hélio Langoni ${ }^{2}$, Daniel da S. Penachio ${ }^{2}$, Juliana C.C. Citadella ${ }^{2}$, Felipe Laurino ${ }^{2}$, \\ Patricia Y. Faccioli-Martins ${ }^{2}$, Simone B. Lucheis ${ }^{3}$, Benedito D. Menozzi ${ }^{2}$ \\ e Aristeu V. da Silva ${ }^{4}$
}

\begin{abstract}
Langoni H., Penachio D.S., Citadella J.C.C., Laurino F., Faccioli-Martins P.Y., Lucheis S.B., Menozzi B.D. \& Silva A.V. 2011. [Quality and microbiological aspects of bovine milk.] Aspectos microbiológicos e de qualidade do leite bovino. Pesquisa Veterinária Brasileira 31(12):1059-1065. Departamento de Higiene Veterinária e Saúde Pública, Faculdade de Medicina Veterinária e Zootecnia, Universidade Estadual Paulista, Botucatu, SP 18618000, Brazil. E-mail: hlangoni@fmvz.unesp.br

Mastitis is the main disease in dairy herds, presents high prevalence and constitutes a limiting factor on many farms in Brazil due to economic losses. Considering the etiological complexity of mastitis the present work aimed to study the etiological agents of mastitis and its influence on the quality of bovine milk. For this, a total of 1090 teats from dairy cows of 10 farms localized in São Paulo state were evaluated. The microbiological analysis of milk consisted of cultivating an $0.1 \mathrm{~mL}$ aliquot of milk from each CMT-positive sample, or with clinical mastitis, on agar base medium containing $5 \%$ of ovine blood and on McConkey agar, incubating plates at $37^{\circ} \mathrm{C}$ with observation of the microbial development at 24-hour intervals during three days. The microorganisms with highest frequency in mastitis were Corynebacterium bovis (29.52\%), Streptococcus dysgalactiae (11.9\%) and Staphylococcus aureus (10.48\%). There was isolation of Candida krusei e Trichosporum spp. on Sabouraud dextrose agar. The averages of SCC and CFU from cows were variabl: eight (80\%) farms were found to be within the limits established by regulation "Instrução Normativa no 51" of the Ministry of Agriculture, and all farms were found to be within the limits for CFU. There was a positive correlation between CFU and SCC from milk in two of six farms statistically analyzed. It was concluded that mastitis is one of the factors that do not allow producer to reach the quality required by the government. Management and hygiene failures exist and must be corrected with instructions for the application of good production practices. Finally, monitoring of mastitis and milk quality in herds must be carried out, and accessible techniques as compound SCC can be used.
\end{abstract}

INDEX TERMS: Milk quality, mastitis, bovine.

RESUMO.- A mastite é a principal afecção do gado leiteiro, possui alta prevalência, e constitui um fator limitante em muitas propriedades rurais do país, devido às perdas

\footnotetext{
${ }^{1}$ Recebido em 31 de março de 2011.

Aceito para publicação em 26 de agosto de 2011.

${ }^{2}$ Departamento de Higiene Veterinária e Saúde Pública, Faculdade de Medicina Veterinária e Zootecnia, Universidade Estadual Paulista (Unesp), Distrito de Rubião júnior, s/n, Botucatu, SP 18618-000, Brasil. *Autor para correspondência: hlangoni@fmvz.unesp.br

${ }^{3}$ Agência Paulista de Tecnologia dos Agronegócios (APTA/SAA), Pólo Centro-Oeste/Unidade de Pesquisa de Bauru, Av. Rodrigues Alves 40, Horto Florestal, Bauru, SP 17030-000, Brasil. E-mail: silucheis@apta.sp.gov.br

${ }^{4}$ Departamento de Ciências Biológicas, Universidade Estadual de Feira de Santana, Rodovia Transnordestina s/n, Novo Horizonte, Feira de Santana, BA 44036-900, Brasil. E-mail: aristeuvsilva@gmail.com
}

econômicas. Considerando-se a complexidade etiológica das mastites o objetivo do presente trabalho foi estudar os agentes de etiológicos desta enfermidade e a sua influência na qualidade do leite bovino. Para tanto, foram avaliados um total de 1090 tetos de animais de dez propriedades rurais localizadas no estado de São Paulo. A análise microbiológica do leite consistiu em cultivar uma alíquota de $0,1 \mathrm{~mL}$ de leite de cada amostra positiva ao CMT, ou com mastite clínica, em meio de ágar base adicionado de 5\% de sangue ovino e em agar Mac Conkey, incubando-se as placas a $37^{\circ} \mathrm{C}$ com observação do desenvolvimento microbiano a cada 24 horas durante três dias. Os microrganismos com maior frequência na mastite foram Corynebacterium bovis $(29,52 \%)$, Streptococcus dysgalactiae $(11,9 \%)$ e Staphylo- 
coccus aureus $(10,48 \%)$. Houve ainda o isolamento em ágar Sabouraud dextrose de Candida krusei e Trichosporum spp. As médias de CCS e UFC dos animais foram variáveis e oito $(80 \%)$ propriedades encontram-se dentro dos limites estabelecidos para CCS pela Instrução Normativa $\mathrm{n}^{\circ} 51$ do Ministério da Agricultura, Pecuária e Abastecimento, e todas as propriedades se encontram dentro dos limites para UFC. Houve correlação positiva entre UFC e CCS de leite em duas propriedades entre as seis analisadas estatisticamente. Conclui-se que a mastite é um dos fatores que não permitem que o produtor atinja a qualidade exigida pelo governo. Falhas de manejo e higiene existem e devem ser corrigidas com treinamento dos produtores para aplicação de boas práticas de produção. Finalmente, o monitoramento das mastites e da qualidade do leite nos rebanhos deve ser realizado, e técnicas acessíveis como a CCS composta podem ser utilizadas.

TERMOS DE INDEXAÇÃO: Qualidade do leite, mastite, bovina.

\section{INTRODUÇÃo}

O agronegócio leite ocupa um espaço de destaque na economia mundial. Este sistema industrial é um dos mais expressivos do Brasil pela sua importância social, e a atividade leiteira é praticada em todo país, em cerca de um milhão de propriedades rurais. Gera mais de três milhões de empregos diretos na produção primária e agrega mais de seis bilhões de reais ao valor da produção agropecuária nacional (Müller 2002).

Com base em dados disponibilizados pela International Dairy Federation (IDF 2005), Guimarães (2006) salientou que o Brasil é o sexto maior produtor de leite. Em 2007 foram produzidos mais de 24 bilhões de litros, o que mostra a importância desta atividade para o país. Costa (2005) ressaltou que a Organização Mundial de Saúde (OMS) recomenda o consumo diário mínimo de 500 mililitros de leite por dia pelo adulto e no mínimo de um litro durante a adolescência e terceira idade. Entretanto, no Brasil, o consumo médio do produto per capita é inferior a um copo diário, cerca de $170 \mathrm{~mL}$.

Paralelamente a sua importância como fonte nutricional indispensável à saúde humana, o leite e seus derivados também são excelentes meios para o desenvolvimento de micro-organismos desejáveis, e outros patogênicos e deteriorantes (Teuber 1992), havendo a necessidade de cuidados desde a sua produção, ordenha, beneficiamento e estocagem. Ruegg (2003) destacou que riscos à saúde humana relacionados ao consumo de leite e derivados, podem decorrer do consumo de produtos lácteos crus, de erros na pasteurização, da contaminação destes por patógenos termorresistentes emergentes e/ou da seleção de agentes de zoonoses resistentes a antimicrobianos, além da sua adulteração.

A mastite é a principal afecção de bovinos destinados à produção leiteira, que impacta negativamente a economia, considerando-se a sua alta prevalência nos rebanhos. As perdas econômicas se devem a vários fatores, tais como a diminuição da produção, custos com mão de obra, honorários profissionais, gastos com medicamentos, morte ou descarte precoce de animais e queda na qualidade do produto final, com diminuição no rendimento industrial (Langoni et al. 1998).

Mais de 140 diferentes tipos de microrganismos podem causar mastite (Philpot \& Nickerson 2002). Entre os patógenos mais frequentes, estão Staphylococcus aureus, Streptococcus agalactiae, Streptococcus dysgalactiae, Streptococcus uberis, Escherichia coli, Corynebacterium bovis, Pseudomonas aeruginosa, Enterobacter spp. e outros, como Mycoplasma spp., Mycobacterium bovis, Brucella abortus e Listeria monocytogenes. (Langoni et al. 1998, Martins et al. 2010).

A contagem de células somáticas (CCS) no leite é uma ferramenta importante no diagnóstico da mastite subclínica, aceita internacionalmente como medida padrão para determinar a qualidade do leite cru e, consequentemente, para monitorar a sanidade da glândula mamária (Harmon 1998, Philpot 1998, Smith \& Hogan 1998, Santos 2006). Podem ser utilizados métodos diretos de contagem celular pelo exame eletrônico em aparelhos como o SOMACOUNT-300 (Bentley ${ }^{\circledR}$ ), ou indiretos, pelo California Mastitis Test (CMT) (Schalm \& Noorlander 1957).

Para melhorar a qualidade do leite e garantir um alimento seguro e de alto valor nutricional, é fundamental o controle da mastite nos rebanhos. Muitos esforços têm sido empregados para o seu controle. Tem-se adotado o estabelecimento de pagamento por qualidade, baseado na redução da contagem de células somáticas por mililitro de leite, que reflete o nível de ocorrência de mastite. No Brasil, o Programa Nacional de Melhoria da Qualidade do Leite (PNMQL), pela Portaria no 51 do Ministério da Agricultura (Brasil 2002), implementada em 2005, prevê a redução da $\mathrm{CCS}$, resfriamento do leite nas propriedades leiteiras, redução da contagem total de micro-organismos, ausência de resíduos químicos, pagamento por qualidade e/ou penalidades para incentivar o controle de mastite (Guimarães \& Langoni 2009).

Considerando-se a importância da obtenção de leite de boa qualidade nas propriedades rurais, objetivou-se com o presente estudo avaliar microbiologicamente a qualidade do leite analisando a CCS e contagem de unidades formadoras de colônias (UFC) verificando a correlação entre os resultados de CCS de leite e UFC.

\section{MATERIAL E MÉTODOS}

Foram examinadas 283 vacas em lactação, primíparas e multíparas, da raça Holandesa e mestiça, procedentes de dez propriedades rurais, de diferentes regiões do Estado de São Paulo, sendo uma de São Pedro, Nova Odessa, Pirassununga, Porto Feliz, três de Paraibuna e três de Botucatu, submetidas à ordenha mecânica ou manual, totalizando 1090 tetos, pois 42 eram afuncionais. Os animais foram examinados antes da ordenha, quanto às características do leite, avaliando-se os primeiros jatos pela prova do Tamis para detecção dos casos clínicos, avaliando-se a presença de grumos, alteração de coloração do leite, presença de pus, sangue ou leite aguado (Blood \& Radostitis 2000).

Realizou-se o exame do California Mastitis Test (CMT) (Schalm \& Noorlander 1957), considerando-se como reações positivas a partir de uma cruz (+). Foram colhidas amostras de leite em frascos estéreis após limpeza do teto, lavagem com água corrente, 
secagem com toalha de papel descartável e utilização de solução de álcool iodado a 5\%, para desinfecção do óstio do teto. Após desprezarem-se os primeiros jatos de leite, foram coletados ao redor de $10 \mathrm{~mL}$ de leite para o exame microbiológico, transportados em caixa de material isotérmico, com gelo reciclável, até ao laboratório, onde as amostras foram processadas.

De cada animal com pelo menos um teto com CMT positivo, colheu-se amostra de leite composta para realização da CCS eletrônica em aparelho SOMACOUNT-300 (Bentley ${ }^{\circledR}$ ), bem como de amostra do tanque da propriedade. Da mesma forma, colheu-se amostra de $50 \mathrm{~mL}$ de leite do tanque, observando-se condições de assepsia, para realização de contagem de unidades formadoras de colônias (UFC), mantendo-se sob temperatura de refrigeração, até o momento do exame. Procedeu-se ainda à contagem de UFC de amostra de leite composta de cada animal, com pelo menos um teto positivo ao CMT, colhendo-se ao redor de $10 \mathrm{~mL}$ de leite de cada teto, após a desinfecção como descrito anteriormente.

Para a contagem de UFC, foram procedidas diluições a $10^{-1}, 10^{-}$ 2 e $10^{-3}$, semeando-se $0,1 \mathrm{~mL}$ de cada diluição, em placa de Petri com meio de ágar-sangue ovino a 5\%, espalhando-se por toda a superfície, com auxílio da alça de Drigalski estéril, com a contagem após 24 horas de incubação a $37^{\circ} \mathrm{C}$. Para a pesquisa da flora aeróbica foram cultivados $0,1 \mathrm{~mL}$ de cada amostra em duplicata, incubando-se por até 96 horas a $37^{\circ} \mathrm{C}$. Os micro-organismos isolados foram corados por Gram e repicados em meio de caldo cérebro coração para realização de provas bioquímicas segundo Krieg \& Holt (1994),Quinn et al. (1994) e Murray et al. (1999). Para identificação de Staphylococcus spp. foram realizadas as provas de catalase e coagulase, e posteriormente os coagulase-positivos foram submetidos a provas de fermentação dos açúcares trealose, maltose e manitol e resistência à polimixina $\mathrm{B}$ (300UI) e para os coagulase-negativos fermentação dos açúcares xilose, sacarose, trealose, maltose, manitol e crescimento em tiogel. Streptococcus spp foram identificados pela realização das provas de catalase, CAMP teste e hidrólise da esculina, enterobactérias pelos meios de EPM, MILi e citrato e Corynebacterium spp. pela urease, fermentação da glicose e maltose. Consideraram-se como positivas as amostras com isolamento de três ou mais colônias de um mesmo micro-organismo, exceto para Staphylococcus aureus, que com o isolamento de uma ou mais colônias era considerada positiva e, como contaminantes, aquelas com o isolamento de três ou mais agentes diferentes (National Mastitis Council 1999).

Para o estudo da flora fúngica, $100 \mu \mathrm{L}$ de cada amostra foram cultivadas em duplicata, com o auxílio de bastão de vidro, em placas de Petri contendo meio de Sabouraud Dextrose Agar (SDA). As colônias leveduriformes isoladas em SDA foram subcultivadas em tubos contendo Agar Saboraund suplementado com extrato de levedura (SDA+EL-peptona 1\%, glicose 4\%, extrato de levedura $1 \%$, Agar $1,5 \%$ ) por 24 horas a $37^{\circ} \mathrm{C}$. A seguir, foram procedidas às provas bioquímicas de auxanograma e zimograma, bem como a produção de tubo germinativo para as espécies do gênero Candida spp (Lacaz 1984).

As frequências de animais segundo o resultado do CMT foram comparadas entre as propriedades pelo teste de $\chi^{2}$. As contagens de células somáticas e de unidades formadoras de colônias foram comparadas entre as propriedades usando-se o teste não-paramétrico de Kruskall-Wallis. Para cada propriedade, a correlação entre a CCS e a UFC foi determinado pelo cálculo do coeficiente de Spearmann. Em todas as análises, valores de P menores que 0,05 foram considerados significativos (Triola 2005).

\section{RESULTADOS}

Os resultados referentes aos exames do CMT e teste de Tamis nas dez propriedades são apresentados no Quadro 1.
Quadro 1. Distribuição dos resultados de mastite subclínica e clínica nas propriedades

\begin{tabular}{|c|c|c|c|c|c|c|c|}
\hline \multirow{3}{*}{$\begin{array}{l}\text { Propriedade } \\
\text { (n- animais) }\end{array}$} & \multirow[t]{3}{*}{ № de tetos } & \multicolumn{4}{|c|}{ Mastite subclínica } & \multirow{2}{*}{\multicolumn{2}{|c|}{$\begin{array}{c}\text { Mastite } \\
\text { clínica }\end{array}$}} \\
\hline & & \multicolumn{2}{|c|}{ CMT negativo } & \multicolumn{2}{|c|}{ CMT positivo } & & \\
\hline & & $\mathrm{N}$ & $\%$ & $\mathrm{~N}$ & $\%$ & $\mathrm{~N}$ & $\%$ \\
\hline $1(40)$ & 148 & 85 & 57,43 & 63 & $42,57^{d}$ & 1 & 0,67 \\
\hline $2(38)$ & 136 & 71 & 52,20 & 65 & $47,79^{d}$ & 0 & 0 \\
\hline $3(37)$ & 140 & 114 & 81,42 & 26 & $18,57^{c}$ & 1 & 0,71 \\
\hline $4(21)$ & 83 & 76 & 91,57 & 7 & $8,43^{\mathrm{a}}$ & 1 & 1,20 \\
\hline $5(35)$ & 139 & 137 & 98,58 & 2 & $1,44^{\mathrm{a}}$ & 0 & 0 \\
\hline 6 (37) & 157 & 146 & 92,99 & 11 & $7,01^{\mathrm{a}}$ & 0 & 0 \\
\hline $7(22)$ & 85 & 68 & 80,00 & 17 & $20,00^{\mathrm{b}}$ & 2 & 2,35 \\
\hline $8(24)$ & 99 & 92 & 92,93 & 7 & $7,07^{\mathrm{a}}$ & 1 & 1,01 \\
\hline $9(15)$ & 52 & 46 & 88,46 & 6 & $11,54^{\mathrm{b}}$ & 0 & 0 \\
\hline $10(14)$ & 51 & 45 & 88,24 & 6 & $11,76^{\mathrm{b}}$ & 2 & 3,92 \\
\hline Total (283) & 1090 & 880 & & 210 & & 8 & \\
\hline
\end{tabular}

Estatística: $\chi^{2}=186,04$, valor de $\mathrm{P}<0,0001$. Valores de positivos seguidos de letras diferentes indicam diferenças significativas entre as propriedades.

Observa-se uma grande diferença na ocorrência de mastite subclínica variando de 1,44 a 47,79\%. As propriedades com CCS elevada ( $>15 \%$ ) foram número 1, 2, 3 e 7 . A ocorrência da mastite clínica variou de 0 a $3,92 \%$, tendo um número de casos elevado na Propriedade 10 (3,92\%).

Como pode ser observado no Quadro 2, houve o isolamento de diversos agentes nas amostras de mastite subclínica, com predomínio de Corynebacterium bovis $(29,52 \%)$, Streptococcus dysgalactiae (11,9\%) e Staphylococcus aureus $(10,48 \%)$, e cultura negativa em 36 (17,14\%) amostras. Nos casos de mastite clínica foram isolados $S$. dysgalactiae, Streptococcus agalactiae e Pasteurella multocida, separadamente, e associações de Klebsiella pneumoniae+Escherichia coli, $S$. aureus $+S$. dysgalactiae, $S$. agalactiae $+S$. dysgalactiae, sendo o agente predominante $S$. dysgalactiae. Duas (25\%) amostras foram negativas ao isolamento.

No Quadro 3 e na Figura 1 podem ser vistos os resultados da análise estatística para CCS dos animais, de acordo com seis propriedades, excluindo-se da análise aquelas com número inferior a oito tetos positivos ao exame microbiológico. Pode-se observar que as Propriedades 1 e 3 apresentaram valores de CCS significativamente inferiores à Propriedade 2. As demais propriedades não apresentaram diferença significante.

Os resultados de UFC das amostras de leite compostas dos animais, de acordo com as propriedades em que foi possível realizar a análise estatística, são visualizados nos Quadro 4 e na Figura 2. Não houve diferença significativa na contagem de UFC entre as Propriedades 1, 2, 3, 4 e 6 mas estas foram inferiores às contagens da Propriedade 7.

No Quadro 5 observa-se a correlação significativa e positiva nas Propriedades 1 e 4.

A CCS dos tanques apresentou-se baixa $(<200.000$ células/mL), exceto na Propriedade 2 que foi de 586.000 células $/ \mathrm{mL}$, tendo esta propriedade o maior valor de CCS composta (870.000 células $/ \mathrm{mL}$ ) e a maior prevalência de mastite subclínica.

Quanto à UFC das amostras de leite dos tanques de expansão, os resultados revelaram o envolvimento de micro-organismos que em seis (60\%) propriedades não foram isolados nas amostras de leite individual examinadas, incluindo Escherichia coli, Klebsiella pneumoniae, Pseudomonas aeruginosa, Candida guilliermondii e Trichosporon spp. 
Quadro 2. Microrganismos isolados de amostras de leite com mastite subclínica e clínica nas propriedades estudadas

\begin{tabular}{|c|c|c|c|c|}
\hline \multirow[t]{2}{*}{ Micro-organismo } & \multicolumn{2}{|c|}{ Mastite subclínica } & \multicolumn{2}{|c|}{ Mastite clínica } \\
\hline & $\mathrm{n}$ & $(\%)$ & $\mathrm{n}$ & $(\%)$ \\
\hline Corynebacterium bovis & 62 & 29,52 & - & - \\
\hline Streptococcus dysgalactiae & 25 & 11,9 & 1 & 12,5 \\
\hline Staphylococcus aureus & 22 & 10,48 & - & - \\
\hline Staphylococcus epidermidis & 15 & 7,14 & - & - \\
\hline Streptococcus agalactiae & 15 & 7,14 & 1 & 12,5 \\
\hline S. aureus $+C$. bovis & 4 & 1,9 & - & - \\
\hline S. dysgalactiae + C. bovis & 3 & 1,43 & - & - \\
\hline Nocardia sp. + C. bovis & 3 & 1,43 & - & - \\
\hline Escherichia coli & 3 & 1,43 & - & - \\
\hline S. hyicus + C. bovis & 2 & 0,95 & - & - \\
\hline S. dysgalactiae + S. uberis & 2 & 0,95 & - & - \\
\hline Streptococcus uberis & 2 & 0,95 & - & - \\
\hline Klebsiella pneumoniae & 2 & 0,95 & - & - \\
\hline S. aureus + S. dysgalactiae & - & - & 1 & 12,5 \\
\hline S. aureus + S. uberis & 1 & 0,48 & - & - \\
\hline S. agalactiae $+S$. dysgalactiae & - & - & 1 & 12,5 \\
\hline S. agalactiae + S.uberis & 1 & 0,48 & - & - \\
\hline S. agalactiae + C. bovis & 1 & 0,48 & - & - \\
\hline S. epidermidis $+S$. uberis & 1 & 0,48 & - & - \\
\hline S. epidermidis $+C$. bovis & 1 & 0,48 & - & - \\
\hline Nocardia sp. & 1 & 0,48 & - & - \\
\hline Nocardia sp. + S. agalactiae & 1 & 0,48 & - & - \\
\hline K. pneumoniae + E. coli & 1 & 0,48 & 1 & 12,5 \\
\hline Pseudomonas aeruginosa & 1 & 0,48 & - & - \\
\hline Pasteurella multocida & 1 & 0,48 & 1 & 12,5 \\
\hline Streptococcus bovis & 1 & 0,48 & - & - \\
\hline Trichosporum spp. + P. aeruginosa & 1 & 0,48 & - & - \\
\hline Candida krusei & 1 & 0,48 & - & - \\
\hline Staphylococcus intermedius & 1 & 0,48 & - & - \\
\hline Cultura negativa & 36 & 17,14 & 2 & 25 \\
\hline TOTAL & 210 & 100 & 8 & 100 \\
\hline
\end{tabular}

Os valores mais elevados da mediana da UFC composta foram das Propriedades 6 (18.000 UFC/mL) e 7 (80.000 $\mathrm{UFC} / \mathrm{mL}$ ) que também apresentaram os valores elevados de UFC do tanque (490.000 e $420.000 \mathrm{UFC} / \mathrm{mL}$ ).

\section{DISCUSSÃO E CONCLUSÕES}

A ocorrência de mastite subclínica foi considerada elevada em quatro (P1, P2, P3 e P7) das dez propriedades, considerando um limite normal de ocorrência de até 15\% no rebanho, segundo Santos \& Fonseca (2007). Essas propriedades possuíam rebanhos selecionados, alta produção, manejo adequado e mecanização da ordenha. Porém esses animais são mais susceptíveis a infecções e a ordenhadeira, se não for bem utilizada e conservada, favorece ainda mais a transmissão de patógenos da mastite entre os animais.

Da mesma forma, uma propriedade (P10) apresentou

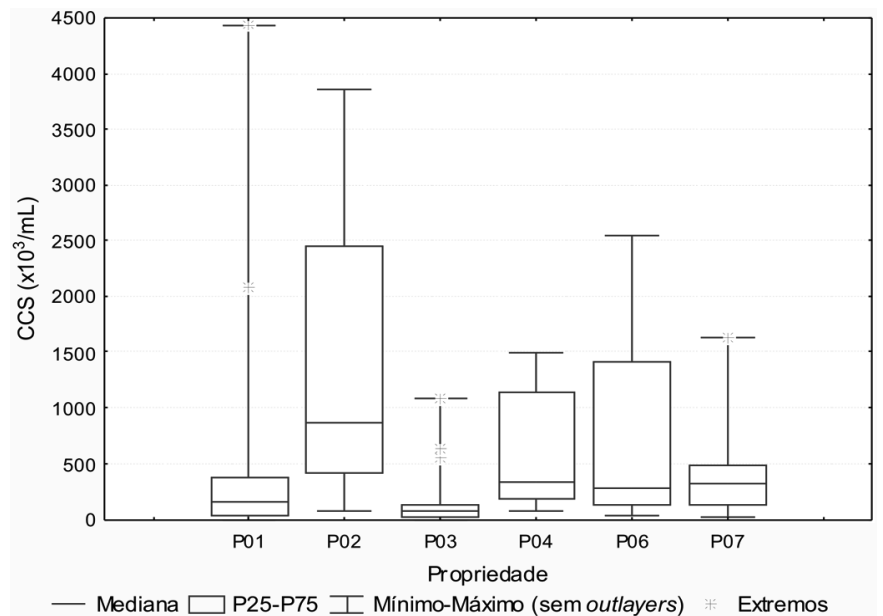

Fig.1. Mediana, percentis 25 (P25) e 75 (P75), valores mínimos e máximos (sem outlayers) e valores extremos da CCS (X103) do leite bovino, de amostras compostas, segundo a propriedade (P).

ocorrência elevada (>2\%) de mastite clínica. 0 manejo nesta propriedade era ruim, com deficiência na higienização do estábulo e a ordenha era mecanizada, justificando o isolamento de agente ambiental em associação com agentes contagiosos (S. aureus e S. agalactiae).

Analisando estudos realizados em outros estados, essas ocorrências de mastite subclínica e clínica foram inferiores às obtidas em Cuiabá/MT, em que se identificaram 64,6\% e 5,8\% amostras de leite como mastite subclínica e clíni$\mathrm{ca}$, respectivamente, foram semelhantes a Teresina/PI com prevalência de $41,1 \%$ de mastite subclínica, e foram inferiores a Rondon do Pará/PA em que se diagnosticou 6,6\% de amostras com mastite subclínica e 1,3\% de mastite clínica (Ferreira et al. 2007, Martins et al. 2010, Oliveira et al. 2011).

Em Cuiabá/MT as propriedades possuíam animais de diferentes raças, ordenha mecânica e deficiências no manejo, na higiene principalmente na desinfecção pós-ordenha, e na terapêutica das mastites. A maior ocorrência de mastites em relação ao presente estudo pode ser justificada pelas propriedades de Cuiabá serem todas tecnificadas, com manejo mais intensivo e maior produção, e da mesma forma com falhas na higiene e na terapêutica das mastites.

Nas propriedades de Teresina/PI os animais eram mestiços, a ordenha era manual, as condições de instalações, higiene e manejo eram inadequadas, e não eram realizadas práticas de controle da mastite, características semelhante

Quadro 3. Estatísticas descritivas da contagem de células somáticas $\left(\mathrm{x10}^{3} / \mathrm{mL}\right)$ de amostras compostas de leite bovino, segundo a propriedade examinada

\begin{tabular}{ccccccccc}
\hline Propriedade & $\mathrm{N}$ & Média & Desvio Padrão & Mínimo & P25 & Mediana & P75 & Máximo \\
\hline P1 & 36 & 418,2 & 805,3 & 0,0 & 41,5 & $164,0^{\mathrm{a}}$ & 374,0 & 4437,0 \\
P2 & 33 & 1386,4 & 1201,3 & 81,0 & 410,0 & $870,0^{\mathrm{b}}$ & 2453,0 & 3863,0 \\
P3 & 21 & 172,8 & 272,4 & 1,0 & 32,0 & $85,0^{\mathrm{a}}$ & 139,0 & 1096,0 \\
P4 & 8 & 610,5 & 555,5 & 82,0 & 209,5 & $330,5^{\text {ba }}$ & 1100,0 & 1485,0 \\
P6 & 8 & 780,3 & 979,0 & 38,0 & 154,0 & $283,5^{\text {ba }}$ & 1084,0 & 2545,0 \\
P7 & 14 & 462,2 & 458,2 & 28,0 & 176,3 & $323,5^{\text {ba }}$ & 497,3 & 1638,0
\end{tabular}

Estatística: valores de mediana seguidos de letras diferentes indicam diferenças significativas entre as propriedades (P), pelo teste não-paramétrico de Kruskall-Wallis (estatística $\mathrm{H}=39,48 ; \mathrm{p}<0,0001)$. 
Quadro 4. Estatística descritiva da contagem de UFC $\left(\mathrm{x10}^{3}\right)$ de leite bovino, de amostras compostas, segundo a propriedade examinada

\begin{tabular}{ccccccccc}
\hline Propriedade & N & Média & Desvio Padrão & Mínimo & P25 & Mediana & P75 & Máximo \\
\hline P1 & 35 & 97.04 & 322.63 & 0.04 & 0.80 & $2.28^{\mathrm{a}}$ & 14.00 & 1480.00 \\
P2 & 33 & 3.49 & 0.48 & 0.05 & 1.60 & $2.90^{\mathrm{a}}$ & 4.70 & 11.00 \\
P3 & 21 & 2.52 & 0.23 & 0.80 & 1.65 & $2.40^{\mathrm{a}}$ & 3.20 & 5.20 \\
P4 & 8 & 4.24 & 2.68 & 1.58 & 2.40 & $3.35^{\mathrm{a}}$ & 5.90 & 9.00 \\
P6 & 8 & 128.04 & 245.73 & 0.70 & 3.80 & $18.00^{\mathrm{a}}$ & 130.00 & 720.00 \\
P7 & 14 & 118.59 & 161.84 & 1.20 & 37.00 & $80.00^{\mathrm{b}}$ & 120.00 & 620.00
\end{tabular}

Estatística: valores de mediana seguidos de letras diferentes indicam diferenças significativas entre as propriedades (P), pelo teste não-paramétrico de Kruskall-Wallis (estatística $\mathrm{H}=24,4921 ; \mathrm{p}=0,0002$ ).

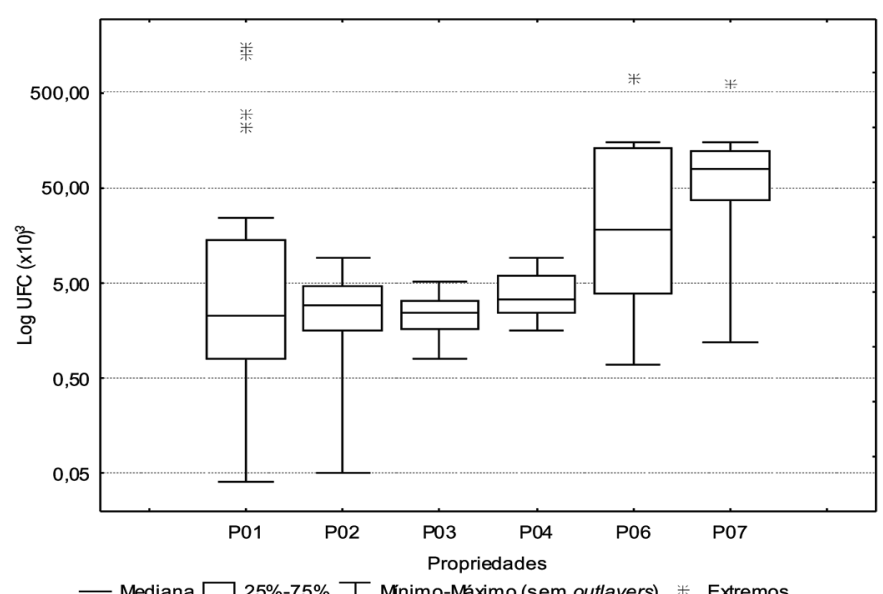

Fig.2. Mediana, percentis 25 (P25) e 75 (P75), valores mínimos e máximos (sem outlayers) e valores extremos da UFC (em escala logarítmica) de leite bovino, segundo a propriedade.

Quadro 5. Coeficientes de correlação entre a CCS e a UFC do leite bovino, segundo a propriedade examinada

\begin{tabular}{ccc}
\hline Propriedade & $\mathrm{R}$ & Valor de P \\
\hline 1 & 0,3775 & 0,0253 \\
2 & 0,1485 & 0,4094 \\
3 & $-0,1109$ & 0,6323 \\
4 & 0,8095 & 0,0218 \\
6 & $-0,0476$ & 0,9346 \\
7 & $-0,2291$ & 0,4308
\end{tabular}

Estatística: valores de P menores que 0,05 indicam correlações significativas pelo cálculo do coeficiente de Spearmann.

observada nas Propriedades 4, 5 e 6. Em Rondon do Pará/ PA os animais eram mestiços com baixa produção leiteira, a ordenha era manual, a higiene da ordenha e do ambiente era precária e não eram realizados testes para diagnóstico da mastite. Essas propriedades apresentaram menores índices de mastite em comparação ao presente estudo provavelmente por praticarem um sistema de criação extensivo, os animais terem baixa produção e pela permanência do bezerro com as vacas, não permitindo o acúmulo de leite residual na glândula mamária.

A diversidade de agentes etiológicos isolados dos casos de mastite também foi observada por outros autores (Rodrigues \& Ruegg 2005, Martins et al. 2010, Oliveira et al. 2011). Martins et al. (2010), assim como no presente estudo, também obtiveram maior prevalência de Corynebacterium spp. $(27,60 \%)$ e $S$. aureus $(21,51 \%)$, e encontraram uma porcentagem semelhante de culturas negativas $(24,37 \%)$ para mastite subclínica e $24,0 \%$ (para mastite clínica). 0 isolamento de Candida krusei e Trichosporon spp. é relatado na literatura. Normalmente está associado à falha na anti-sepsia, tratamento intramamário repetitivo ou higiene precária da ordenhadeira (Santos \& Marin 2005, Costa et al. 2008, Gaudie et al. 2009).

Avaliando-se o perfil das propriedades, observa-se que P1, P2 e P3 que possuem um bom manejo, têm gado puro e ordenha mecânica, os agentes predominantes são os contagiosos (Corynebacterium bovis, Staphylococcus spp.) e uma porcentagem de ambientais, provavelmente por falhas na higienização dos tetos e por excesso de sujidades nos piquetes ou camas (Streptococcus dysgalactiae, Trichosporon spp.). As Propriedades 4, 5 e 6 que possuem gado mestiço, manejo ruim e ordenha manual apresentam uma microbiota mista de agentes ambientais (Nocardia sp., S. dysgalactiae) e contagiosos (S. aureus, S. agalactiae). A Propriedade 7 possui um rebanho mestiço mas bem manejado e com ordenha mecânica, predominando uma microbiota contagiosa (S. agalactiae, Corynebacterium bovis) e Nocardia sp. como ambiental. As Propriedades 8 e 9 também possuem gado mestiço mas com manejo inadequado e ordenha mecânica, apresentando tanto agentes contagiosos (S. agalactiae, Corynebacterium bovis, S. aureus, S. hyicus) como ambientais (S.dysgalactiae, Klebsiella pneumoniae, Escherichia coli). Por fim, a Propriedade 10 que possui gado mestiço, manejo inadequado e ordenha mecânica apresentou microbiota contagiosa (Corynebacterium bovis, S. aureus, $S$. agalactiae).

Quanto à mediana da CCS composta dos animais, é possível verificar que P2 tem o valor mais alto, justificado pela sua alta prevalência de mastite subclínica no rebanho e pelo isolamento de agentes contagiosos que estimulam o aumento da CCS (Rainard \& Riollet 2006). As Propriedades 4, 6 e 7 também tiveram mediana da CCS superior a 200.000 células $/ \mathrm{mL}$, o que concorda com a literatura quanto a esse valor ser o limite para determinar se um animal está com mastite. Além disso, os resultados de CCS de amostras compostas são os que têm maior significado, por serem os mais utilizados pelos produtores em programas de controle de mastite, e serem um bom indicador da ocorrência de infecções intramamárias (Santos 2006). A mediana da UFC composta dos animais foi menor em P1, P2 e P3, pois realizam um melhor controle da mastite no rebanho.

Considerando a CCS média dos animais de cada propriedade, frente ao limite determinado até julho de 2011 ( $<750.000$ células $/ \mathrm{mL}$ ) pela Instrução Normativa no 51 do Ministério da Agricultura, Pecuária e Abastecimento, 
observa-se que oito $(80 \%)$ propriedades estariam em conformidade. Se for considerado o limite em vigor a partir de julho de 2011 ( $<400.000$ células/mL), somente uma propriedade estaria adequada. Para a UFC média dos animais com o limite até julho de 2011, todas as propriedades estariam em conformidade, porém com o limite reduzido a partir de julho de 2011 ( $<100.000 \mathrm{UFC} / \mathrm{mL}$ ) duas propriedades estariam inadequadas. Com esses dados é possível verificar que a mastite nos animais pode afetar drasticamente a qualidade do leite do tanque, e que se um monitoramento do rebanho e um controle das mastites não forem realizados periodicamente, os produtores não terão condições de atingir as metas estabelecidas pelo governo e não fornecerão um leite de qualidade ao consumidor.

$\mathrm{Na}$ análise de correlação da CCS composta com a UFC composta, observaram-se duas propriedades com correlação positiva, o que demonstra que houve o aumento de CCS com o aumento da UFC. Na Propriedade 1 isso se justifica pela alta prevalência de mastite subclínica, causada pelo elevado número de bactérias no leite e com a resposta à infecção houve aumento da CCS. Mesmo com uma menor prevalência de mastite subclínica, tal fato ocorreu também com a Propriedade 4.

A CCS do tanque, segundo a literatura e como se observou na P2, também é uma medida indireta do percentual de quartos mamários infectados no rebanho e apresenta relação positiva com a prevalência da mastite no rebanho. Não se esquecendo de que a predominância de um tipo de agente causador (ambiental ou contagioso) tem efeito significativo sobre a CCS do tanque (Santos 2006).

Quanto à UFC de leite das amostras dos tanques de expansão, o isolamento de micro-organismos não presentes nas amostras de leite individuais indica contaminação ambiental do leite durante e após a ordenha. As Propriedades 6 e 7 que apresentaram os valores mais elevados da mediana da UFC composta foram as que apresentaram os valores mais elevados de UFC do tanque, demonstrando mais uma vez o impacto da mastite na qualidade final do leite.

Conclui-se, portanto, que a mastite ainda é um problema importante nos rebanhos leiteiros por afetar a saúde dos animais, reduzir a produção e causar alterações significativas na qualidade do leite, sendo um dos fatores que não permitem que os produtores atinjam as metas determinadas pelo governo. Uma situação prejudicial para o produtor por receber descontos no preço do leite, para a indústria por ter o tempo de prateleira reduzido e para o consumidor final que não tem acesso a um produto de qualidade.

Algumas falhas de manejo dos animais e de ordenha têm ocorrido nas propriedades e a contaminação do leite do tanque com micro-organismos ambientais tem sido observada. Ressalta-se, desta forma, a necessidade de treinamento adequado e constante dos produtores para aplicação de boas práticas de produção.

Por fim, a CCS e UFC compostas trouxeram informações úteis sobre a situação do rebanho, em destaque a CCS composta que pode ser utilizada na prática das propriedades para avaliar a condição dos animais e do leite produzido.

Agradecimentos.- À FAPESP, pelo suporte financeiro de bolsa de Iniciação Científica (Proc.2006/07133-2).

\section{REFERÊNCIAS}

Blood D.C. \& Radostitis O.M. 2000. Veterinary Medicine: a textbook of the disease of cattle, sheep, pigs, goats and horses. $9^{\text {th }}$ ed. W.B. Saunders, London. 1877p.

Brasil 2002. Instrução Normativa no 51, de 20 de setembro de 2002, Ministério da Agricultura. Diário Oficial da União, Brasília, DF, Seção 1, p.13.

Costa E.O. 2005. Programa nacional de melhoria da qualidade do leite (PNMQL). Napgama 8:18-21.

Costa G.M., Silva N., Rosa C.A., Figueiredo H.C.P. \& Pereira U.P. 2008. Mastite por leveduras em bovinos leiteiros do Sul do Estado de Minas Gerais, Brasil. Ciência Rural 38:1938-1942.

Ferreira J.L., Lins J.L.F.H.A., Cavalcant T.V., Macedo N.A. \& Borjas A.L.R. 2007. Prevalência e etiologia da mastite bovina no município de Teresina, Piauí. Ciênc. Anim. Bras. 8:261-266.

Gaudie C.M., Wragg P.N. \& Barber A.M. 2009. Outbreak of disease due to Candida krusei in a small dairy herd in the UK. Vet. Rec. 165:535-537.

Guimarães F.F. 2006. Modificação na geografia da produção mundial de leite. Napgama 9:19-23.

Guimarães F.F. \& Langoni H. 2009. Leite: alimento imprescindível, mas com riscos para a saúde pública. Vet. Zootec. 16:38-51.

Harmon R.J. 1998. Somatic cell counts: myths vs reality. Proc. $37^{\text {th }}$ National Mastitis Council Regional Meeting, Bellevue, Madison, p.40-50.

International Dairy Federation 2005. The World Dairy Situation. Bulletin of the IDF no.399/2005, The International Dairy Federation (IDF), Brussels. 86p.

Krieg N.R. \& Holt J.C. 1994. Bergey's Manual of Sistematic Bacteriology. $9^{\text {th }}$ ed. Williams and Wilkins, Baltimore. 984p.

Lacaz C.S. 1984. Micologia médica: fungos, actinomicetos e algas de interesse médico. Sarvier, São Paulo. 479p.

Langoni H., Silva A.V., Cabral K.G. \& Domingues P.F. 1998. Aspectos etiológicos na mastite bovina: flora bacteriana aeróbica. Revta Bras. Med. Vet. 20:204-209.

Martins R.P., Silva J.A.G., Nakazato L., Dutra V. \& Almeida Filho E.S. 2010. Prevalência e etiologia infecciosa da mastite bovina na microrregião de Cuiabá, MT. Ciênc. Anim. Bras. 11:181-187.

Muller E.E. 2002. Qualidade do leite, células somáticas e prevenção da mastite. Simpósio sobre Sustentabilidade da Pecuária Leiteira na Região Sul do Brasil, Anais II Sul-Leite, Toledo, PR, p.206-217.

Murray P.R., Baron E.J., Pfaller M.A., Tenover F.C. \& Yolken R.H. 1999. Manual of Clinical Microbiology. $7^{\text {th }}$ ed. American Society of Microbiology, Washington. 1773p.

National Mastitis Council 1999. Laboratory Handbook on Bovine Mastitis. The National Mastitis Council (NMC), Arlington. 222p.

Oliveira C.M.C., Sousa M.G.S., Silva N.S., Mendonça C.L., Silveira J.A.S., Oaigen R.P., Andrade S.J.T. \& Barbosa J.D. 2011. Prevalência e etiologia da mastite bovina na bacia leiteira de Rondon do Pará, estado do Pará. Pesq. Vet. Bras. 31:104-110.

Philpot W.N. 1998. Importância da contagem de células somáticas e outros fatores que afetam a qualidade do leite. Anais Simpósio Internacional sobre Qualidade do Leite, Curitiba, p.28-35.

Philpot W.N. \& Nickerson S.C. 2002. Vencendo a Luta contra a Mastite. Westfalia Landtechnik do Brasil Ltda, p.189.

Quinn P.J., Carter M.E., Markey B. \& Carter G.R. 1994. Clinical Veterinary Microbiology. Wolfe Publishing, London. 648p.

Rainard P. \& Riollet C. 2006. Innate immunity of the bovine mammary gland. Vet. Res. 37:369-400.

Rodrigues A.C.O. \& Ruegg P.L. 2005. Actions and outcomes of Wisconsin dairy farms completing milk quality teams. J. Dairy Sci. 88:2672-2680.

Ruegg P.L. 2003. Practical food safety interventions for dairy production. J. Dairy Sci. 86(Suppl.): E1-E9.

Santos M.V. 2006. 0 uso da CCS em diferentes países, p.181-197. In: Ibid. (Ed.), Perspectivas e Avanços da Qualidade do Leite no Brasil. Talento, Goiânia. 352p. 
Santos M.V. \& Fonseca L.F.L. 2007. Estratégias para controle de mastite e melhoria da qualidade do leite. Manole, Barueri. 314p.

Santos R.C. \& Marin J.M. 2005. Isolation of Candida spp. from mastitic bovine milk in Brazil. Mycopathologia 159:251-253.

Schalm G.N. \& Noorlander D.D. 1957. Experiments and observations leading to development of the California Mastitis Test. J. Am. Med. Assoc. 130:199-204.
Smith K.L. \& Hogan J.S. 1998. Milk quality, a worldwide perspective. Proc. $37^{\text {th }}$ National Mastitis Council Annual Meeting, St Louis. National Mastitis Council, Madison, p.3-9.

Teuber M. 1992. Microbiological problems facing the dairy industry. Bull. 276, International Dairy Federation, p.6-9.

Triola M.F. 2005. Introdução à Estatística. 9a ed. LTC, Rio de Janeiro. $682 \mathrm{p}$. 